\title{
Dosimetric comparison of left-sided whole breast irradiation with 3D-CRT, IP-IMRT and hybrid IMRT
}

\author{
XIAOXUE XIE $^{1 *}$, SHUYU OUYANG $^{1 *}$, HUI WANG $^{1}$, WENJUAN YANG $^{1}$, \\ HEKUN JIN $^{1}$, BINGQIANG HU ${ }^{1}$ and LIANGFANG SHEN ${ }^{2}$ \\ ${ }^{1}$ Department of Radiation Oncology, Hunan Provincial Tumor Hospital and Affiliated Tumor Hospital of Xiangya \\ Medical School, Central South University, Changsha, Hunan 410013; ${ }^{2}$ Department of Radiation Oncology, \\ Xiangya Hospital, Central South University, Changsha, Hunan 410008, P.R. China
}

Received August 6, 2013; Accepted September 30, 2013

DOI: $10.3892 /$ or.2014.3058

\begin{abstract}
The aim of this study was to compare the dosimetric characteristics of left-sided whole breast irradiation among 3-dimensional conformal radiotherapy (3D-CRT), 4-field inverse-planned intensity-modulated radiotherapy (IP-IMRT) and hybrid IMRT technique (combining 3D-CRT beams and IP-IMRT beams) with respect to target coverage and irradiation of organs at risk. The 3 different planning techniques were analyzed for 8 patients with left-sided breast conserving surgery. Plans were compared on the basis of planning target volume (PTV) dose conformity, homogeneity and the volumes of normal tissues treated based on dose-volume histograms (DVHs). DVHs were calculated for the PTV, heart, and the bilateral lungs, contralateral breast, and soft tissue surrounding the breast PTV $\left(\mathrm{V}_{\mathrm{OB}}\right)$ volume. IP-IMRT and hybrid IMRT techniques comparably improved the PTV dose homogeneity and conformity (CI) significantly, compared to the conventional 3D-CRT technique $(\mathrm{P}<0.017)$; the IP-IMRT technique only could additionally benefit patients by decreasing the high-dose (40 Gy) volume for heart and ipsilateral lung compared with the hybrid IMRT technique $(\mathrm{P}<0.017)$; the hybrid IMRT plans achieved a further improvement by compromising the increase of low-dose volume (total lung V13, contralateral lung V5, heart V10 and soft tissue surrounding the breast V5) compared with IP-IMRT plans $(\mathrm{P}<0.017)$. Hybrid IMRT plans achieved equivalent PTV dose uniformity to IP-IMRT plans and compromised the low-dose volume and requirement of clinic resource between IP-IMRT and 3D-CRT plans, promoting it as a standard practice of left-sided breast irradiation for patients in good-ordered cardiopulmonary health.
\end{abstract}

Correspondence to: Dr Liangfang Shen, Department of Radiation Oncology, Xiangya Hospital, Central South University, 87 Xiangya Road, Changsha, Hunan 410008, P.R. China

E-mail: oddsnowwhite@gmail.com

${ }^{*}$ Contributed equally

Key words: whole breast irradiation, intensity-modulated radiotherapy, hybrid intensity modulated radiotherapy

\section{Introduction}

Breast conservation surgery is initially performed on patients suffering from breast cancer. Thereafter, radiotherapy of the whole breast is performed on these patients as it is the standard mode of treatment. Treatment is mostly executed using a wedge-based 3-dimensional conformal technique. In this case, 2 opposing tangential fields are chosen to target the entire breast. However, care is taken to minimize the exposure to lung tissues within the treatment fields. In clinical practice, wedges are frequently employed to determine the differential thickness across the breast, while blocks or static multileaf collimators (MLCs) are strategically placed to shield the heart and lung as much as possible. The conventional 3-dimensional conformal radiotherapy (3D-CRT) has been successful in improving local control $(1,2)$. However, wedges can only provide 2-dimensional compensation of missing tissue, which could be suboptimal and result in an inhomogeneous dose distribution, particularly in the case of women with large breasts (3). Large breast size often deters homogeneous results in increased hot spots: these are located within both the target and the surrounding normal tissues. Previous studies have suggested that dose variations $>5-10 \%$ of the prescribed dose within the target breast (hot spots) correlated with the occurrence of soft-tissue toxicity. However, these measures were associated with poor cosmetic outcomes (4). Moreover, the concave shape of the chest wall and overlying breast results in unavoidable irradiation to portions of the underlying lung and heart with 3D-CRT. This is particularly true while treating patients diagnosed with cancer in the left breast (5). In addition, physical compensators significantly scatter the dose to the contralateral breast (6). In this case, the patient becomes more vulnerable to developing radiation-induced contralateral breast cancer (7). Thus, normal tissue toxicities remain an area of critical concern $(1,8,9)$.

Intensity-modulated radiation therapy (IMRT) has been used in breast cancer treatment. IMRT boosts improvements in dose distribution to the target volume, while minimizing the exposure of high volume doses to heart and lung tissues (12). In other words, it helps in achieving the objective of reducing acute and late radiation toxicity $(10,11)$. Thus, IMRT has emerged as a standard treatment of several inflicted sites (13). 
Delivery of irradiation to an irregular shape can be optimized with IMRT. In addition, the technology offers the ability to produce concavities in the treatment volume so as to improve conformality. Studies in breast cancer patients have shown that better dose uniformity is achieved throughout the breast with IMRT, wherein a median of $0.1 \%$ of the treatment volume receives $\geq 110 \%$ of the prescribed dose vs. $10 \%$ with conventional wedges (14). However, IMRT has an important shortcoming; it increases the volume of low-dose exposure for major normal organs due to its dose distribution characteristics. It is important to reiterate that this fact has already been confirmed in previous studies, which investigated the dose distribution of IMRT used in the treatment of other diseases (15-18). Furthermore, this technique requires longer planning and treatment times. Apart from this, an additional time is required for processing the quality assurance (QA) of the IMRT beams. A significant workload of the radiotherapy department has been associated with the execution of adjuvant breast cancer radiotherapy. Thus, a slight increase in the treatment complexity will have serious implications for resource allocations. IMRT has been associated with substantially greater costs. Thus, it is financially cumbersome for the patient (or insurance company). Hence, while determining the most appropriate RT for a patient, one must also consider resource limitations (19).

Mayo et al (20) found that the 4-field hybrid IMRT plan (combining 2 open tangents with tangential IMRT beams), with a quality comparable to that of forward-planned IMRT (FP-IMRT), could be achieved in substantially less planning time. Moreover, improvements in the uniformity of dose to the target volume and conformality may be achieved by adding 2 anterior oblique IMRT beams to the 4-field hybrid technique (6-field hybrid IMRT). In a clinical trial conducted by Farace et al (21), hybrid IMRT was performed by direct aperture optimization. However, when treatment goals were not achieved by using a 4 -field technique, a 6 -field technique was applied. Their results proved that hybrid IMRT can be planned for a large number of patients with little impact on human or departmental resources. Here, hybrid IMRT is an improvised version that is executed with the help of semi-automated tools. Mayo et al (20) compared the dose distribution of 2-field tangent-only IMRT plans with hybrid IMRT. They propounded that the 2 -field tangent-only IMRT plans were more effective in reducing the high-dose exposure to lungs and heart. However, these plans worsened dose homogeneity within the breast. Moreover, they also worsened the maximal dose outside the target. Under such circumstances, 2 open fields are essential to achieve requisite dose uniformity for treating breasts afflicted with cancer. Multi-beam IMRT can reduce the high-dose region outside the planning target volume (PTV) and reach homogeneity dose distribution inside the PTV simultaneously. Under such conditions, open fields are not necessary (22). However, we need to determine whether a hybrid IMRT planning technique can achieve dosimetric equivalence comparable to that of multi-beam IMRT, particularly in cases of patients with cancer detected in the left breast. However, the comparison between hybrid IMRT and multibeam IMRT has rarely been reported in medical literature.

In this study, we compared the efficacy of different oncology techniques, such as 3D-CRT, 4-field IP-IMRT and hybrid IMRT (combining 3D-CRT and 4-field IP-IMRT). We examined these techniques based on 2 important parameters: dose to the PTV and organs at risk (OAR). In this study, we deliberately restricted our analysis to the IMRT and hybrid plans as they employ the same tangential beam angles that are used for the 3D-CRT plan. This analysis explores the dose directed to the normal tissue and the associated exit dose through heart and lungs (23).

\section{Materials and methods}

Patient preparation. Eight patients were randomly selected for this dosimetric study. These patients were diagnosed with left-sided breast cancer. They had previously been treated with breast conserving surgery by a single oncologist at Xiangya Hospital. The breast volumes for these selected patients varied between 304 to $1633 \mathrm{cc}$, with an average breast volume of $812.75 \pm 444.93 \mathrm{cc}$. These values were generally encountered in breast cancer patients. The patients were immobilized in a vacuum pad; CT scans were performed on these patients in the treatment position, marking the range of breast with lead wires. Scans were transferred to the treatment planning computer (Varian Eclipse version 6.7; Varian Medical Systems, Palo Alto, CA, USA). The breast tissue [clinical target volume, (CTV)] was defined by a radiation oncologist. The contralateral breast, right lung, left lung, and heart tissues were delineated in the CT scans.

Volume delineation. The breast CTV was delineated by the radiation oncologist with the following considerations. According to anatomic references, the CTV is generally defined superiorly by the inferior aspect of the clavicular head and inferiorly by the inframammary fold. This is identified through skin reconstruction and physical examination. Medially, the CTV is limited by the sternum and is generally delineated by $2 \mathrm{~cm}$, which is medial to the edge of the sternum. Laterally, the breast tissue is identified in the mid-axillary line. While dealing with different cases of breast cancer, physicians can make exceptions to these anatomic references after performing a physical examination and image assessment.

A planning volume, the PTV was defined to extend beyond the CTV by as much as $1 \mathrm{~cm}$ in the superior and inferior direction, $0.5 \mathrm{~cm}$ in other directions, and then modified to exclude $0.5 \mathrm{~cm}$ of the buildup region near the skin. This additional margin pushes the high-dose gradient farther away from the edge of the PTV. Thus, in actual PTV coverage, the effect of day-to-day variability gets reduced in patient setup. Excluding the region near the skin drives the optimization algorithm away from attempting to achieve full dose in the buildup region. After completion of optimization on the IMRT PTV, normalization of the plan was performed by referring the coverage of breast PTV.

The body was delineated on the CT scans, and Boolean operations were used to construct a modified body volume that excluded breast PTV. Dose-volume histograms (DVHs) of this tissue outside the breast PTV $\left(\mathrm{V}_{\mathrm{OB}}\right)$ were used to characterize doses associated with non-target tissue within the radiation fields.

Treatment planning techniques. Three treatment plans were developed for each patient. These plans were executed, and 

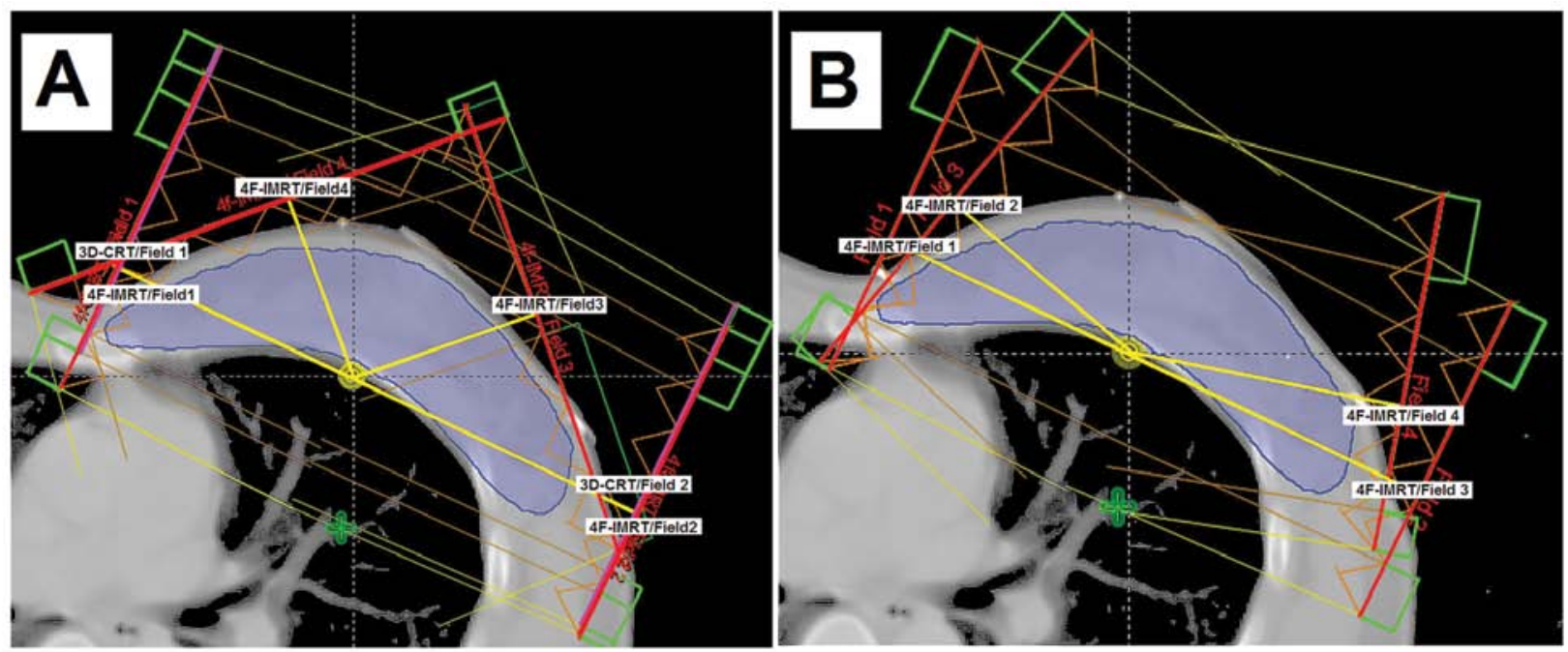

Figure 1. Beam arrangements of (A) hybrid plan and (B) IMRT plan are shown for 1 patient. 3D-CRT, 3-dimensional conformal radiation therapy; IMRT, intensity-modulated radiation therapy.

the afflicted breast was exposed to 50 Gy using $6 \mathrm{MV}$ photon beams. While executing the plan on each patient, the same isocenter and tangential beams were applied. All plans were developed to suit the requisites of a $6 \mathrm{MV}$ beam on a $2100 \mathrm{C} / \mathrm{D}$ accelerator. Millennium 80 MLC from Varian was used as the instrument. In clinical practice, the following methods are routinely used in treating breast cancer patients.

The 3D-CRT plan consisted of standard medial and lateral tangent beams with wedges. MLCs were used to shield the heart and lung tissues. According to the requirements, $15-30^{\circ}$ physical wedges and dynamic wedges were used to treat patients.

The IMRT plan consisted of 4-IMRT fields. These IMRT beams were focused at angles, with an objective of reducing hot spots created outside the breast tissue, especially in the entrance regions of the tangent beams. IMRT beams were $\sim 15^{\circ}$ anterior from the nearest tangent beams (Fig. 1A).

The hybrid IMRT plan combined 2 3D-conformal beams and 4-IMRT beams. The standard medial and lateral primary beam MLCs were designed for the 3D-CRT, but they were used without wedges. These IMRT beams were focused at angles that aimed to reduce hot spots created outside the breast tissue, especially in the entrance regions of the tangent beams. Thus, these beams were $\sim 45^{\circ}$ anterior from the nearest tangent beams (Fig. 1B). The relative weights of the 3D-conformal beams and IMRT beams calculated by the optimization algorithm were acceptable; $40 \%$ of the dose was delivered with IMRT beams.

The IMRT-involved plans were normalized, and the prescribed dose (50 Gy) was received by at least $90 \%$ of the breast PTV. As shown in Table I, these normalized plans were developed using the Varian Medical Systems Eclipse/Helios treatment planning system with the optimization constraints. Optimization was stopped when these constraints were forced beyond permissible limits; the PTV dose homogeneity was compromised. After optimization, the intensity profiling was carried out in the anterior direction such that it extended up to $2 \mathrm{~cm}$ beyond the skin surface; this provided adequate margin for the patient's breathing and accommodated set-up uncertainties.
Table I. Optimization parameters used in Eclipse/Helios in this study for all plans involving intensity-modulated radiotherapy.

\begin{tabular}{llr}
\hline Tissue limit & Limit & Dose $(\mathrm{Gy})$ \\
\hline PTV & Max & $<55$ \\
& Min & $>45$ \\
& $\mathrm{D}_{90 \%}$ & $>50$ \\
Ipsilateral lung & Mean & $<15$ \\
& $\mathrm{D}_{75 \%}$ & $<30$ \\
Contralateral lung & Mean & $<2.5$ \\
Heart & $\mathrm{D}_{95 \%}$ & $<40$ \\
& $\mathrm{D}_{90 \%}$ & $<30$ \\
Contralateral breast & $\mathrm{Mean}$ & $<1.0$ \\
\hline
\end{tabular}

PTV, planning target volume.

Plan evaluation criteria. Batho power law correction was used to evaluate tissue heterogeneity during dose calculations. Isodose contour distributions of different plans were evaluated and compared. Cumulative DVHs were evaluated to assess target volumes and normal structures. Quantitative data were extracted from the DVHs. Plans were compared through 3 significant parameters: PTV dose conformity, homogeneity, and the volumes of normal tissues treated.

To evaluate the quality of plans implemented in the treatment of tumors, the conformity index (CI) and heterogeneity index (HI) were computed on the basis of DVHs of PTVs. CI was defined as the product of the fraction of PTV receiving at least Dmin and the ratio of the volume of PTV receiving at least Dmin to the volume of tissue receiving at least Dmin (the treated volume, $\left.\mathrm{V}_{\mathrm{t}}\right)$. Thus, $\mathrm{CI}=\left(\mathrm{V}_{\mathrm{PTV} 95 \%} / \mathrm{V}_{\mathrm{PTV}}\right) \times\left(\mathrm{V}_{\mathrm{PTV} 95 \%} / \mathrm{V}_{\mathrm{t}}\right)$. A larger $(<1) \mathrm{CI}$ indicated a greater volume of overlap between PTV and treated volume, suggesting that the plan was able to achieve better dose conformity in the treatment. HI was defined using the equation $\mathrm{HI}=\mathrm{D} 5 \% / \mathrm{D} 95 \%$, where D5\% and 

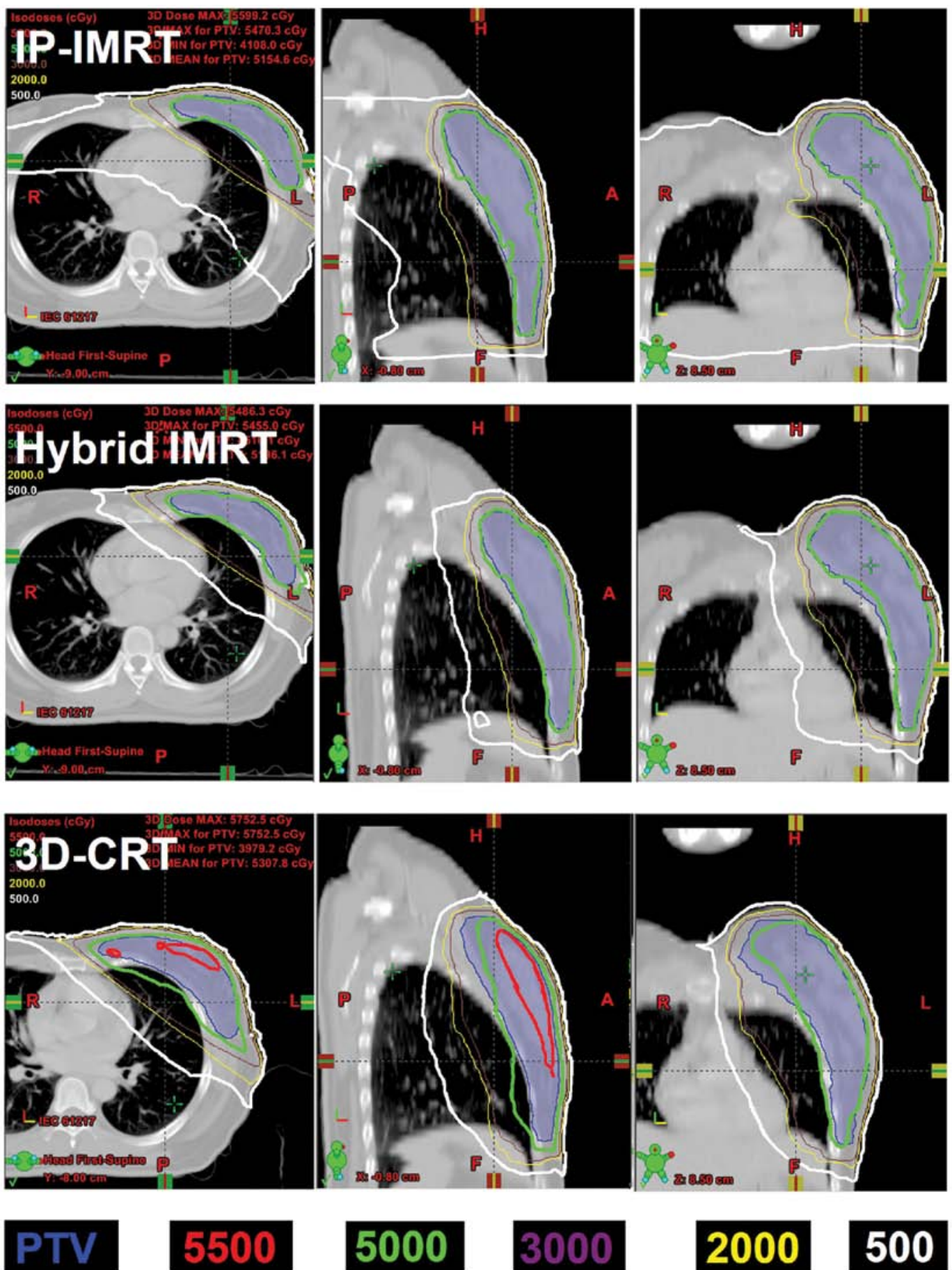

Figure 2. Typical isodose distribution plane for 3D-CRT, IP-IMRT, and hybrid IMRT. 3D-CRT, 3-dimensional conformal radiation therapy; PTV, planning target volume; IMRT, intensity-modulated radiation therapy; HI, inhomogeneity index.

D95\% correspond to the dose given to 5 and $95 \%$ of the PTV, respectively. A smaller $(>1) \mathrm{HI}$ indicated that a smaller dose exceeded the prescription dose, thereby suggesting the prevalence of better dose homogeneity inside the PTV.

We compared the normal tissues treated on the basis of the following parameters: heart max dose and volume receiving 30 Gy or greater (V30), ipsilateral (left) lung mean dose and volume receiving 20 Gy or greater (V20), and contralateral (right) lung mean dose and volume receiving 5 Gy or greater (V5). The primary goal of the IMRT plans was to reduce the volume of exposure to heart and lung, while the patient received a high RT dose. The parameters selected for comparison of heart (V30) and ipsilateral lung (V20, V13) were chosen, as there was evidence $(42,48)$ to prove that doses beyond these values could cause acute or late clinical symptoms. To assist further analysis, V40, V20, V10 and V5 parameters were recorded for heart assessment, while V40, V30 and V5 were recorded to assess ipsilateral lung. Soft tissue surrounding the breast and contralateral breast comparison parameters (mean doses and V5) were taken into consideration while determining doses that may be associated with a carcinogenic risk. A major goal of these plans was to reduce hot spots in the soft tissue 
surrounding the breast. These regions were investigated by evaluating the volume of tissue outside the breast PTV $\left(\mathrm{V}_{\mathrm{OB}}\right)$ receiving 100 and $110 \%$ of the prescribed dose.

Total monitor units (MUs) were tabulated for each plan. The ratio for each plan of total MUs to those for the 3D-CRT plan was calculated for each patient.

Statistical analysis. A two-way analysis of variance (ANOVA) was performed without replication in order to determine if significant differences existed among the planning techniques. Thereafter, paired t-tests were used to identify which techniques differed from others for each dosimetric parameter. To compare the 3 techniques, there were 3 comparisons of each parameter. To determine statistical significance, a two-sided significance level of $\mathrm{P}<0.017$ was used by taking into account Bonferroni's correction (24).

\section{Results}

Fig. 2 displays the typical results for the isodose distributions in each of the 3 plans analyzed in this study. The 3D-CRT technique reduced the volume of tissue irradiated outside the breast by using MLCs to conform the radiation fields. However, a large volume of tissue outside the breast was still encompassed (shown as the green line in Fig. 2), especially in patients having a thorax with a larger curvature. These hot spots are generally located where the physical thickness is less (shown as the red line in Fig. 2), i.e., they are usually located near the lungs (due to their low density compared to surrounding tissue), the apex of the breast, and the axilla. Here, the patient is radio-graphically 'thinner' than on the central axis. The IMRT-involved plans (4-field IP-IMRT and hybrid plans) markedly reduced the hot regions and ensured a more conformal dose distribution around the breast tissue. However, the IMRT plan increased the low-dose exposure on the volume of tissues outside the breast (shown as the white line in Fig. 2).

Fig. 3 represents a typical comparison of DVHs among the 3D-CRT, IP-IMRT and hybrid plans. Compared with the IP-IMRT plans, the relative volume of breast receiving $>100 \%$ prescribed dose was considerably larger when patients were treated with the 3D-CRT plans. By contrast, compared with the IP-IMRT plans, the relative volumes of low-dose region in vital organs (heart, lungs and contralateral breast) were considerably smaller in the 3D-CRT plans. Compared with the 3D-CRT and IP-IMRT plans, hybrid IMRT achieved a good balance between the inner hot spots and low-dose region outside the breast PTV.

As shown in Fig. 4, compared with the 3D-CRT plan, both IMRT and hybrid plans showed an improvement in dose characteristics of the PTV. The relative volume of PTV $>110 \%$ dose (hot spots) was significantly reduced in the IMRT-involved plans. On the other hand, the relative volume of PTV $>95 \%$ and PTV $>90 \%$ dose were significantly greater in hybrid IMRT plans $(\mathrm{P}<0.017)$. As shown in Table II, dose homogeneity was measured by HI while dose conformity was measured by $\mathrm{CI}$. Compared with 3D-CRT plans, both these parameters showed significant improvements through IMRT-involved plans. Improved dose homogeneity was achieved through hybrid techniques. However, this improvement was insignificant with that achieved by the 4-field IP-IMRT plan $(\mathrm{P}=0.024)$.
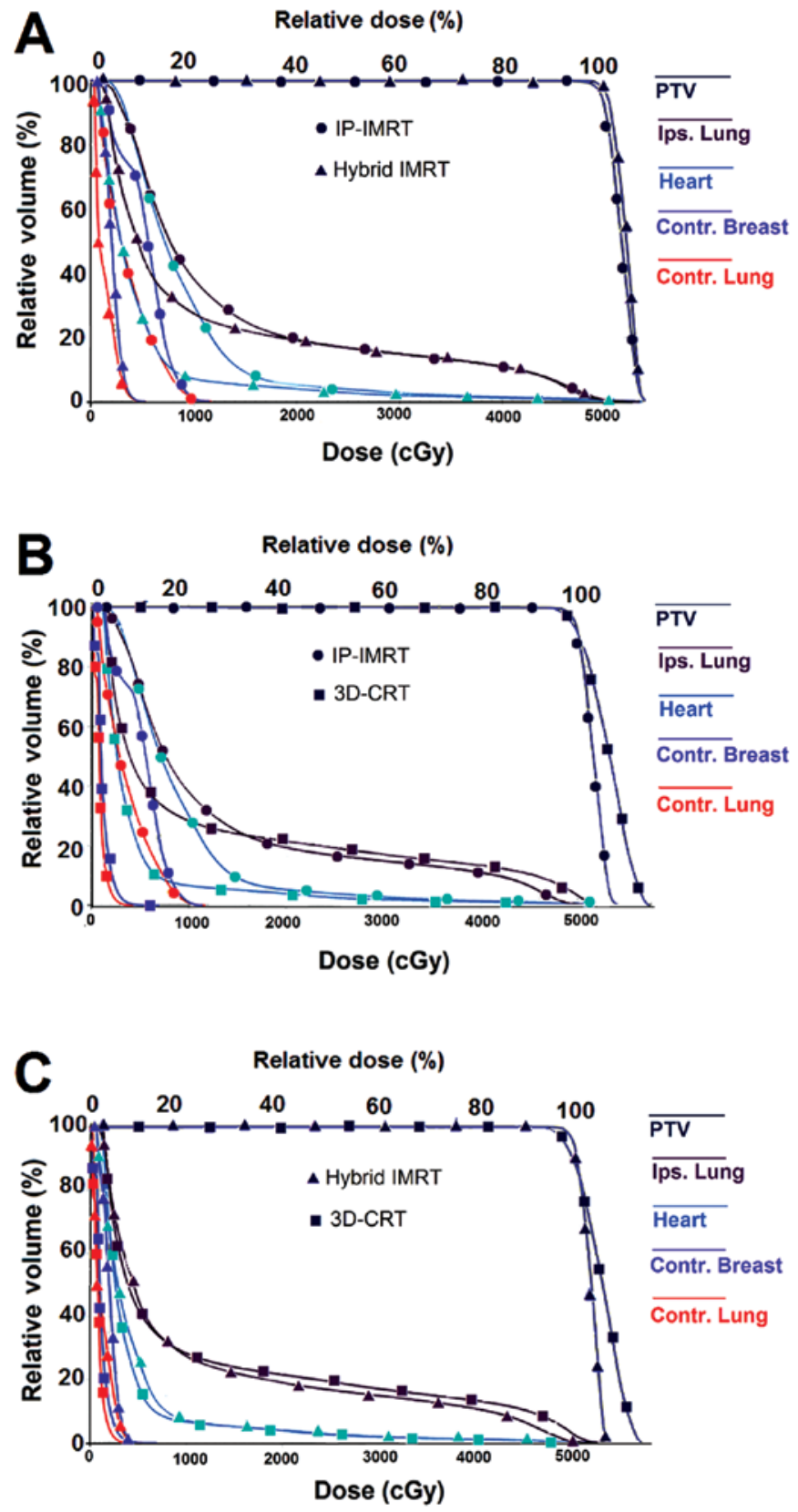

Figure 3. Comparison of typical DVHs for 3D-CRT, IP-IMRT and hybrid IMRT. DVHs, dose-volume histograms; 3D-CRT, 3-dimensional conformal radiation therapy; PTV, planning target volume; IMRT, intensity-modulated radiation therapy; $\mathrm{HI}$, inhomogeneity index.

The average total MUs for 3D-CRT was 319.88 $\pm 36.08 \mathrm{MU}$. Our results illustrate that IP-IMRT plans and hybrid plans require higher average MUs: their MUs were 2.2 and 1.75-fold greater than the 3D-CRT plans, respectively $(\mathrm{P} \leq 0.017)$. This observation of MUs was recorded while delivering the same prescribed dose through different techniques.

Table III displays the characteristics of the dose afflicted to the lungs in each of the techniques. In the case of IMRTinvolved plans, mean dose to the bilateral lungs was significantly higher than that reported for the 3D-CRT plan. In the case of the contralateral lung, the IMRT-involved plans significantly increased the volume receiving $>5$ Gy (V5) as compared to the 3D-CRT plan. In addition, the V5 for the IMRT plan was $>3$-fold higher than that for the hybrid plan. In none of the 
Table II. Dose characteristics of the PTV (breast) associated with the 3 planning strategies, including the mean dose and dose homogeneity for left-sided patients.

\begin{tabular}{lccccccc}
\hline Technique & Mean PTV dose $(\%)$ & $\mathrm{V}_{90}(\%)$ & $\mathrm{V}_{95}(\%)$ & $\mathrm{V}_{100}(\%)$ & $\mathrm{V}_{110}(\%)$ & $\mathrm{HI}$ & $\mathrm{CI}$ \\
\hline 3D-CRT & $104.73 \pm 1.41$ & $99.6 \pm 0.37$ & $98.36 \pm 0.60$ & $90.93 \pm 0.55$ & $9.31 \pm 10.46$ & $1.12 \pm 0.03$ & $0.58 \pm 0.10$ \\
IMRT & $103.88 \pm 0.70$ & $99.56 \pm 0.30$ & $97.92 \pm 0.94$ & $90.82 \pm 0.35$ & $0.18 \pm 0.03^{\mathrm{a}}$ & $1.09 \pm 0.02^{\mathrm{a}}$ & $0.68 \pm 0.10^{\mathrm{a}}$ \\
Hybrid & $103.43 \pm 0.56$ & $99.87 \pm 0.20^{\mathrm{ab}}$ & $99.3 \pm 0.49^{\mathrm{ab}}$ & $92.51 \pm 1.80$ & $0.16 \pm 0.41^{\mathrm{a}}$ & $1.07 \pm 0.01^{\mathrm{a}}$ & $0.66 \pm 1.42^{\mathrm{a}}$ \\
\hline
\end{tabular}

3D-CRT, 3-dimensional conformal radiation therapy; PTV, planning target volume; IMRT, intensity-modulated radiation therapy; HI, inhomogeneity index. ${ }^{\mathrm{a}} \mathrm{P}<0.05$ when compared with $3 \mathrm{D}-\mathrm{CRT}$; ${ }^{\mathrm{b}} \mathrm{P}<0.017$ when compared with $3 \mathrm{D}-\mathrm{CRT}$.

Table III. Characteristics of dose directed to the lungs in different treatment techniques.

\begin{tabular}{llcccccc}
\hline Organ & Technique & Mean dose $(\%)$ & $\mathrm{V}>5 \mathrm{~Gy}(\%)$ & $\mathrm{V}>13 \mathrm{~Gy}(\%)$ & $\mathrm{V}>20 \mathrm{~Gy}(\%)$ & $\mathrm{V}>30 \mathrm{~Gy}(\%)$ & $\mathrm{V}>40 \mathrm{~Gy}(\%)$ \\
\hline Ipsilateral lung & 3D-CRT & $20.06 \pm 1.93$ & $28.82 \pm 2.98$ & $19.39 \pm 1.54$ & $17.26 \pm 1.53$ & $14.5 \pm 2.04$ & $12.34 \pm 2.04$ \\
& IMRT & $22.58 \pm 1.53^{\mathrm{a}}$ & $44.94 \pm 3.38^{\mathrm{a}}$ & $27.66 \pm 2.90^{\mathrm{a}}$ & $17.80 \pm 1.07$ & $13.56 \pm 1.12$ & $10.54 \pm 1.50^{\mathrm{a}}$ \\
& Hybrid & $22.22 \pm 1.23^{\mathrm{a}}$ & $44.35 \pm 7.45^{\mathrm{a}}$ & $20.70 \pm 1.08^{\mathrm{b}}$ & $17.71 \pm 1.32$ & $14.84 \pm 1.44$ & $12.02 \pm 1.36^{\mathrm{b}}$ \\
Contralateral lung & 3D-CRT & $1.77 \pm 0.44$ & $0.45 \pm 1.01$ & $0.27 \pm 0.06$ & $0.00 \pm 0.00$ & $0.00 \pm 0.00$ & $0.00 \pm 0.00$ \\
& IMRT & $2.80 \pm 1.17^{\mathrm{a}}$ & $8.01 \pm 8.33^{\mathrm{a}}$ & $0.01 \pm 0.01$ & $0.00 \pm 0.00$ & $0.00 \pm 0.00$ & $0.00 \pm 0.00$ \\
& Hybrid & $3.48 \pm 0.80^{\mathrm{a}}$ & $2.25 \pm 3.13^{\mathrm{b}}$ & $0.07 \pm 0.19$ & $0.00 \pm 0.00$ & $0.00 \pm 0.00$ & $0.00 \pm 0.00$ \\
\hline
\end{tabular}

3D-CRT, 3-dimensional conformal radiation therapy; PTV, planning target volume; IMRT, intensity-modulated radiation therapy; HI, inhomogeneity index. ${ }^{\mathrm{a}} \mathrm{P}<0.017$ when compared with $3 \mathrm{D}-\mathrm{CRT}$; ${ }^{\mathrm{b}} \mathrm{P}<0.05$ when compared with $3 \mathrm{D}-\mathrm{CRT}$.
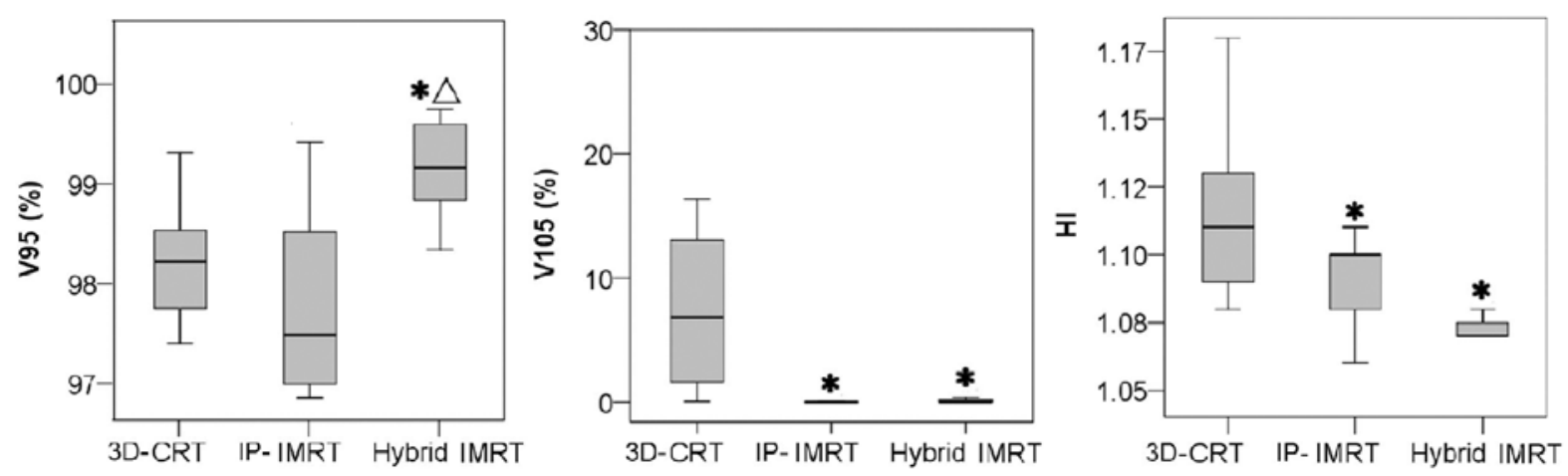

Figure 4. Dose characteristics of the PTV (breast) associated with the 3 planning strategies, including the mean dose and dose homogeneity for left-sided patients. 3D-CRT, 3-dimensional conformal radiation therapy; PTV, planning target volume; IMRT, intensity-modulated radiation therapy; HI, inhomogeneity index. ${ }^{*} \mathrm{P}<0.017$ when compared with $3 \mathrm{D}-\mathrm{CRT} ;{ }^{\wedge} \mathrm{P}<0.017$ when compared with IP-IMRT.

plans did the contralateral lung receive $>20 \mathrm{~Gy}$. In the case of ipsilateral lung, in the low-dose region, the percentage of volume receiving $>5$ Gy was significantly higher for the IMRT-involved plans than for the 3D-CRT plan. Furthermore, the percentage of volume receiving $>13$ Gy was higher in the case of the IMRT plan $(\mathrm{P}<0.017)$. Compared with the other plans, in the high-dose region, the IMRT plan significantly reduced the percentage of ipsilateral lung receiving $>40 \mathrm{~Gy}$. As shown in Fig. 5, while analyzing the entire lung, there was only a slight difference among the 3 techniques with respect to V20: all techniques reported $<8 \%$, but V13 was significantly increased in IP-IMRT plans.
While treating patients diagnosed with cancer in the left breast, the portions of the heart were treated to $>30 \mathrm{~Gy}$, irrespective of the selected plan. However, as shown in Fig. 6, the 3 techniques do not show any significant difference in the following parameters: maximum heart doses and average volume of heart receiving $>30 \mathrm{~Gy}$. In the low-dose region, the percentage of heart receiving $>5$ Gy was significantly greater when patients were subjected to the IMRT-involved plans. For the IMRT plan, the average volume of heart receiving $>10$ Gy was higher, but the average volume of heart receiving $>40$ Gy was significantly lower as compared to the other 2 plans $(\mathrm{P}<0.017)$. 

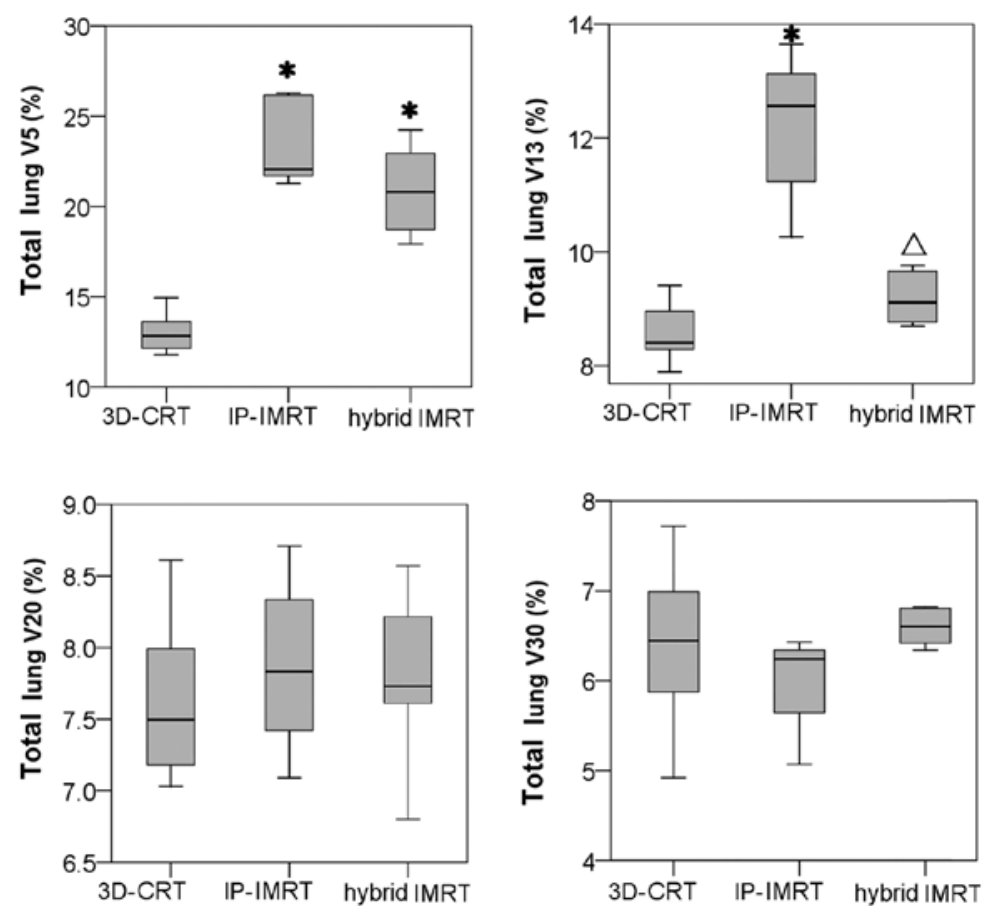

Figure 5. Characteristics of the dose to total lung for each of the treatment techniques. 3D-CRT, 3-dimensional conformal radiation therapy; PTV, planning target volume; IMRT, intensity- modulated radiation therapy; HI, inhomogeneity index. ${ }^{*} \mathrm{P}<0.017$ when compared with $3 \mathrm{D}-\mathrm{CRT}$; ${ }^{\wedge} \mathrm{P}<0.017$ when compared with IP-IMRT.
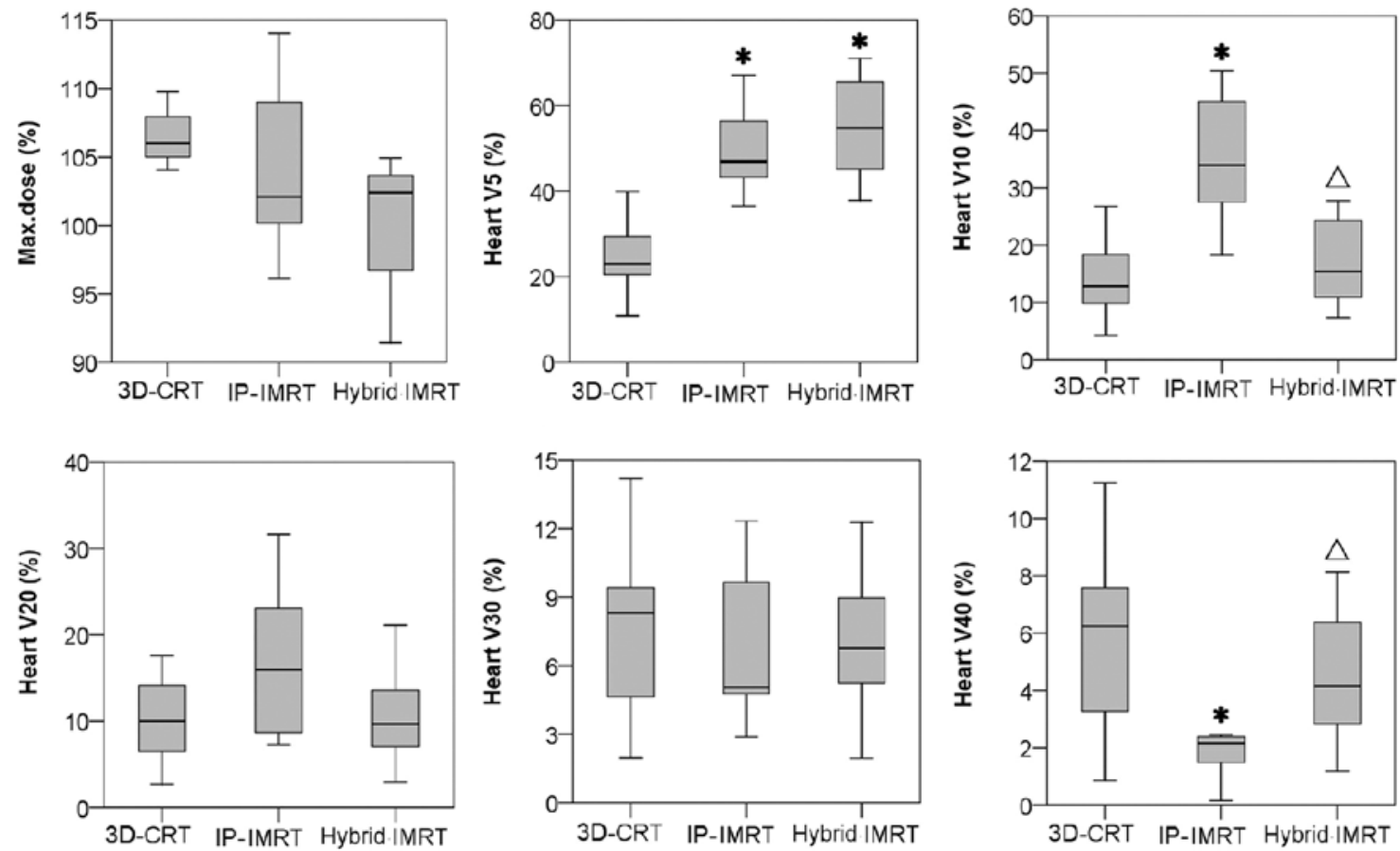

Figure 6. Characteristics of the dose to heart for each of the treatment techniques. 3D-CRT, 3-dimensional conformal radiation therapy; PTV, planning target volume; IMRT, intensity-modulated radiation therapy; HI, inhomogeneity index. ${ }^{*} \mathrm{P}<0.017$ when compared with $3 \mathrm{D}-\mathrm{CRT}$; ${ }^{\triangle} \mathrm{P}<0.017$ when compared with IP-IMRT.

As presented in Fig. 7A, the average mean dose to the contralateral breast was significantly greater for the IMRT plan compared to the other 2 plans. In the case of the IMRT and the hybrid plans, the percentage of contralateral breast receiving $>5$ Gy was significantly higher. Furthermore, the IMRT plans witnessed greater exposure compared to the hybrid plans, but this was statistically insignificant $(\mathrm{P}=0.025)$.
The percentage of contralateral breast receiving $>10$ Gy was reported to be the highest while executing the IMRT plans. The exposure was comparatively less in the case of the other treatment plans, including the hybrid IMRT plans $(\mathrm{P}=0.018)$ and the 3D-CRT plans $(\mathrm{P}=0.018)$. However, this disparity in the parameters of the three treatment plans was statistically insignificant. 

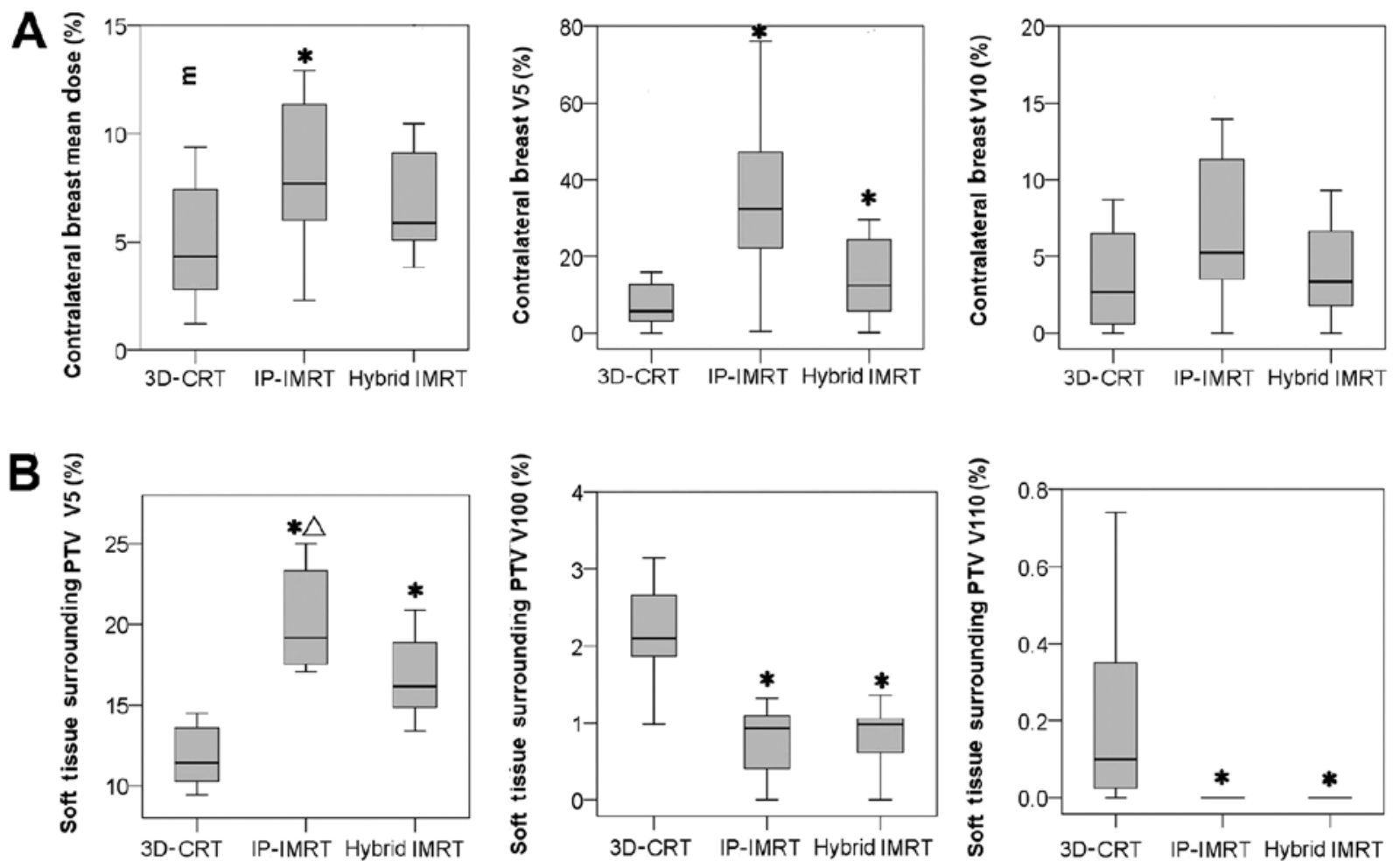

Figure 7. Characteristics of the dose to contralateral breast and soft tissue surrounding breast PTV for each of the treatment techniques. 3D-CRT, 3-dimensional conformal radiation therapy; PTV, planning target volume; IMRT, intensity-modulated radiation therapy; HI, inhomogeneity index. ${ }^{*} \mathrm{P}<0.017$ when compared with 3D-CRT; ${ }^{\triangle} \mathrm{P}<0.017$ when compared with IP-IMRT.

Fig. 7B summarizes comprehensively the volume of soft tissue outside the PTV $\left(\mathrm{V}_{\mathrm{OB}}\right)$, which received prescribed or higher dose. Compared to the 3D-CRT technique, the IMRTinvolved plans reduced the volume of tissue that received prescribed dose $\left(\mathrm{V}_{100 \%}\right)$. Similar results were obtained for higher dose $\left(\mathrm{V}_{110 \%}\right)$, and there was 0 volume of tissue receiving $>110 \%$ in the IMRT-involved plans. In the low-dose region, the percentage of VOB receiving $>5$ Gy was significantly greater for the IMRT-involved plans than for the 3D-CRT plan. However, this type of radiation exposure was compromised in the hybrid plan as compared to the IMRT plan ( $\mathrm{P}=0.012)$.

\section{Discussion}

In the present study, the selected patients were representative of the different types of cases encountered in clinical practice. In this case, all reported plans were calculated by taking into account dose heterogeneity. The wedge-based 3D-CRT plans were considered as the standard that optimized the dose uniformity to the target tissue. The inverse-planned IMRT plans were developed on the Varian Eclipse system with the help of Helios optimization algorithm. As anticipated, the improvement in dose distribution was greater in cases of patients with larger breasts, whose hot spot regions encompassed larger areas while treating with 3D-CRT plans.

The dose uniformity to the PTV was comparable for all techniques in our study, wherein the mean doses varied between 103.43 and $104.73 \%$. However, compared with the 3D-CRT technique, the PTV volume receiving high-dose (110\%) witnessed a sharp decline from 9.3 to $0.2 \%$ provided patients were treated with two IMRT-involved techniques. Comparing $\mathrm{HI}$ and $\mathrm{CI}$ among three techniques, the IMRT-involved techniques showed a significant improvement over the 3D-CRT plans. Noticeably, for hybrid IMRT, the PTV volume received 95 or $90 \%$ prescription dose: this was significantly higher than for the other 2 techniques. Thus, compared with the IP-IMRT technique, the hybrid IMRT technique improved HI; however, the improvement was not statistically significant $(\mathrm{P}=0.024)$. The hot spots outside the target were also evaluated. The hybrid IMRT plans were comparable with the IP-IMRT plans, but they were better than the 3D-CRT plans in restricting the volumes of tissue outside the target from receiving $\geq 100$ and $\geq 110 \%$, prescribed dose.

These results indicate that greater dose homogeneity and conformity were achieved by the IMRT-involved plans. While performing IMRT of the breast (25-29), clinical benefits associated with skin toxicity reduction have been reported. Furthermore, it has also been reported that IMRT is beneficial in reducing chronic breast edema (27) and the incidence of change in breast appearance (30). Besides cosmetic requirements, disease control is of paramount importance. However, only 2 studies $(31,32)$ reported on the following outcomes in patients treated for breast cancer: there seemed to be no evidence for differences in local recurrence rates, with less follow-up for patients receiving IMRT-based treatment. This may be attributed to the short follow-up period observed in some studies.

Both IMRT and the hybrid plan had total monitor units (MUs) that were $\sim 2.2$-fold larger than those employed in the 3D-CRT plan. Moreover, the average ratio of total MUS for 
the IMRT plan was significantly higher than for the hybrid plan. Increasing the number of MUs for dynamic deliveries consequently increases the peripheral dose and whole body dose. In fact, it may also increase the probability of radiationinduced secondary malignancies.

When IMRT plans are employed with the field directions that are the same as those encountered with standard tangential irradiation, patients may experience several benefits in the treatment procedures. These benefits are associated with dose reduction to surrounding organs at risk. Nevertheless, additionally improved dose homogeneity within the target volume can only be achieved through more complex IMRT treatments (33-36). In our study, we first proved that hybrid IMRT plans requiring less MUs can achieve dosimetric equivalence to 4-field IP-IMRT. These hybrid IMRTs are also more flexible in terms of positioning repeatability. In the case of hybrid IMRT, the dosimetric equivalence is achieved by combining the same amount of IMRT beams to the standard tangential conformal beams. In this manner, hybrid IMRT plans not only reduce hot spots outside the target volume but also improve dose homogeneity within the target volume.

Heart and lung are the primary organs of concern. In our study, for the IP-IMRT technique, the relative volume of ipsilateral lung or heart receiving high-dose (40 Gy) was significantly reduced. However, the relative volume of tissue receiving low-dose (13 Gy to total lung or $10 \mathrm{~Gy}$ to heart) significantly increased, as compared to the other 2 techniques. With the multi-beam IMRT technique, the relative volume of bilateral lungs and heart receiving even lower dose (5 Gy) comparatively increased while employing IP-IMRT and hybrid techniques. Moreover, the relative V5 of contralateral lung was significantly larger for IP-IMRT than for the hybrid plan. Thus, compared with IP-IMRT, the hybrid technique cannot achieve equivalent reduction in exposure of lung or heart volumes to high-doses ( $>40 \mathrm{~Gy}$ ). However, it does contribute to compromising low-dose volumes. Among all these techniques, the V20 and V30 of the total lung and heart were comparable with each other. Moreover, the max doses to heart were also comparable for all 3 techniques. While devising a planning approach, the physician's clinical judgment plays an important role in deciding how to balance the risks of low-dose levels against high-dose levels.

In clinical practice, we have come across cases of radiation-induced heart disease, wherein the patients' heart partially received therapeutic doses of approximately $\geq 35$ Gy (37). Recent research studies were performed on atom bomb survivors. These studies also suggested a relationship between low-radiation doses in the range of $\leq 4 \mathrm{~Gy}$ and cardiac mortality (38-41). It is reported that 1 Gy added to the mean heart dose could increase the cardiotoxic risk by $4 \%$ (42). The complex process of radiation-induced heart disease involved different heart structures with different radiosensitivities. Under these circumstances, we have not been able to successfully decipher the associated pathomechanisms in patients $(43,44)$. Furthermore, pre-existing cardiovascular risk factors, such as smoking, hypertension, obesity, and the use of cardiotoxic agents generally instigate the development of radiation-induced heart disease. In view of the potential risks, it has been recommended that all measures should be used to reduce radiation exposure to cardiac tissues (43).
Radiation pneumonitis is a rare complication of breast RT, affecting $\sim 1 \%$ of patients receiving breast irradiation (45). In a research study exploring the efficacy of chemoradiation therapy in the treatment of esophageal cancer, Lee et al (46) indicated the importance of volume of lung tissue receiving at least $10 \mathrm{~Gy}$. They illustrated that a similar incidence of complications was reported in cases where $>40 \%$ of lung tissues received at least $10 \mathrm{~Gy}$ and $>20 \%$ of lung received at least $20 \mathrm{~Gy}$. It has been confirmed that total lung V20 is an independent predictor of pneumonitis in lung cancer patients (47). Schallenkamp et al (48) also noted that intrathoracic radiotherapy should be planned cautiously, especially while using radiotherapy techniques delivering doses of 10-5 Gy to large lung volumes. In our study, total lung V20 was $<8 \%$ and comparable for all techniques; total lung V13 significantly increased in the case of the IP-IMRT plans: this increase was $\sim 3 \%$ as compared to the 3D-CRT and hybrid IMRT plans.

While treating breast cancer patients, the dose to the contralateral breast is of paramount significance. In the cases of women diagnosed with breast cancer, the risk of contralateral breast cancer is estimated to be within 2-11\% (49). However, we have not yet been able to comprehend the association with low-dose irradiation. In our plans, for the hybrid technique, the mean dose of the contralateral breast was comparable with the 3D-CRT technique, but it was significantly lower than that for IP-IMRT. The contralateral breast V5 for the hybrid technique was comparable with IP-IMRT, but it was significantly greater than that for the 3D-CRT technique. In summary, hybrid IMRT plans compromised the mean dose and low-dose volume of contralateral breast as compared with the 3D-CRT and IP-IMRT plans.

Whole body dose may be an area of concern; however, we have limited data to estimate the associated risk. Hall and Wuu (50) reported that vulnerability to developing secondary cancer after 10 years may increase in $1 \%$ of patients subjected to conventional radiation therapy and $1.75 \%$ patients subjected to IMRT. In this study, comparing the 3 techniques, the relative volume of soft tissue surrounding the breast receiving at least $5 \mathrm{~Gy}$ was the largest for IP-IMRT (20.25\%) and the smallest for 3D-CRT $(11.8 \%)(\mathrm{P}<0.017)$. Similarly, hybrid IMRT plans compromised the VOB receiving low-dose irradiation between 3D-CRT and IP-IMRT only plans.

As compared to 3D-CRT plans, the hybrid IMRT and IP-IMRT plans were comparable as they improved dose homogeneity and conformity. Owing to these techniques, we could also witness a significant reduction in the magnitude of hot regions outside the target. However, the first trade-off is that these techniques increased low-dose radiation to surrounding areas, including the lungs, heart, contralateral breast and the soft tissue surrounding breast. However, we do not yet know if these increases in low-dose exposure translate into long-term complications or induction of secondary cancer. However, as long as the primary aim of treatment is not affected, these low-dose regions should be minimized. Another trade-off, of course, is the additional time required to perform quality assurance on the IMRT beams. Hence, we need to carefully select patients who would appreciably benefit from IMRT beams; it will also help us in mitigating the impact on clinical resources. 
As compared to the IP-IMRT plans, the hybrid IMRT plans compromised the increase of low-dose volume; however, they promoted the increase of high-dose ( $40 \mathrm{~Gy}$ ) volume in important normal tissues. We need to strike a balance between the risks of low-dose levels against high-dose levels. This plays a pivotal role in influencing the clinical selection of a proper planning technique. In general, multi-beam IMRT plans may benefit elder patients or those patients with cardiopulmonary insufficiency by preventing the heart and lungs from being exposed to high-dose irradiation. Hybrid IMRT may benefit patients in good cardiopulmonary health as the hybrid technique not only improves the cosmetic outcome but also reduces the volume of healthy tissue receiving low-dose irradiation.

In conclusion, compared to the conventional 3D-CRT technique, both the IP-IMRT and hybrid IMRT techniques improved the PTV dose uniformity and conformity. Compared to the hybrid IMRT and the 3D-CRT plans, the IP-IMRT plans achieved significant reduction in the volume of heart and ipsilateral lung exposed to high-dose ( $\geq 40 \mathrm{~Gy}$ ). In general, the multi-beam inverse planned IMRT technique probably benefits patients whose cardiopulmonary condition is poor. Hybrid IMRT plans allowed a PTV coverage and dose homogeneity as good as IP-IMRT only plans. Moreover, it also compromised the low-dose volumes of normal tissues and the demanding clinic resource for IP-IMRT only plans. Therefore, hybrid IMRT plans were preferred as the standard practice for left-whole-breast irradiation. However, no evidence was found for differences in disease control. Furthermore, an additional time was required for executing IMRT-involved plan quality assurance. In conclusion, the 3D-CRT technique should not be excluded as a good selection technique, especially for patients with relatively smaller curvature of thorax, smaller breast volume, and good-ordered cardiopulmonary health.

\section{Acknowledgements}

This study was supported by grants from the National Natural Science Foundation of China (grant no. 81301942).

\section{References}

1. Fisher B, Anderson S, Bryant J, Margolese RG, Deutsch M, Fisher ER, Jeong JH and Wolmark N: Twenty-year follow-up of a randomized trial comparing total mastectomy, lumpectomy, and lumpectomy plus irradiation for the treatment of invasive breast cancer. N Engl J Med 347: 1233-1241, 2002.

2. Veronesi U, Cascinelli N, Mariani L, Greco M, Saccozzi R, Luini A, Aguilar M and Marubini E: Twenty-year follow-up of a randomized study comparing breast-conserving surgery with radical mastectomy for early breast cancer. N Engl J Med 347: 1227-1232, 2002.

3. Das IJ, Cheng CW, Fein DA and Fowble B: Patterns of dose variability in radiation prescription of breast cancer. Radiother Oncol 44: 83-89, 1997.

4. Back M, Guerrieri M, Wratten C and Steigler A: Impact of radiation therapy on acute toxicity in breast conservation therapy for early breast cancer. Clin Oncol 16: 12-16, 2004.

5. Schubert LK, Gondi V, Sengbusch E, Westerly DC, Soisson ET, Paliwal BR, Mackie TR, Mehta MP, Patel RR, Tomé WA and Cannon GM: Dosimetric comparison of left-sided whole breast irradiation with 3DCRT, forward-planned IMRT, inverseplanned IMRT, helical tomotherapy, and topotherapy. Radiother Oncol 100: 241-246, 2011.

6. Kelly CA, Wang XY, Chu JC and Hartsell WF: Dose to contralateral breast: a comparison of four primary breast irradiation techniques. Int J Radiat Oncol Biol Phys 34: 727-732, 1996.
7. Unnithan $\mathrm{J}$ and Macklis RM: Contralateral breast cancer risk. Radiother Oncol 60: 239-246, 2001.

8. Gyenes G, Rutqvist LE, Liedberg A and Fornander T: Long-term cardiac morbidity and mortality in a randomized trial of pre- and postoperative radiation therapy versus surgery alone in primary breast cancer. Radiother Oncol 48: 185-190, 1998.

9. Bird BR and Swain SM: Cardiac toxicity in breast cancer survivors: review of potential cardiac problems. Clin Cancer Res 14: 14-24, 2008

10. Donovan E, Bleakley N, Denholm E, Evans P, Gothard L, Hanson J, Peckitt C, Reise S, Ross G, Sharp G, Symonds-Tayler R, Tait D and Yarnold J; Breast Technology Group: Randomized trial of standard 2D radiotherapy (RT) versus intensity modulated radiotherapy (IMRT) in patients prescribed breast radiotherapy. Radiother Oncol 82: 254-264, 2007.

11. Pignol JP, Olivotto I, Rakovitch E, Gardner S, Sixel K, Beckham W, Vu TT, Truong P, Ackerman I and Paszat L: A multicenter randomized trial of breast intensity-modulated radiation therapy to reduce acute radiation dermatitis. J Clin Oncol 26: 2085-2092, 2008.

12. Beckham WA, Popescu CC, Patenaude VV, Wai ES and Olivotto IA: Is multibeam IMRT better than standard treatment for patients with left-sided breast cancer? Int J Radiat Oncol Biol Phys 69: 918-924, 2007.

13. Selvaraj RN, Beriwal S, Pourarian RJ, Lalonde RJ, Chen A, Mehta K, Brunner G, Wagner KA, Yue NJ, Huq SM and Heron DE: Clinical implementation of tangential field intensity modulated radiation therapy (IMRT) using sliding window technique and dosimetric comparison with 3D conformal therapy (3DCRT) in breast cancer. Med Dosim 32: 299-304, 2007.

14. Kestin LL, Sharpe MB, Frazier RC, Vicini FA, Yan D, Matter RC, Martinez AA and Wong JW: Intensity modulation to improve dose uniformity with tangential breast radiotherapy: initial clinical experience. Int J Radiat Oncol Biol Phys 48: 1559-1568, 2000.

15. Mundt AJ, Mell LK and Roeske JC: Preliminary analysis of chronic gastrointestinal toxicity in gynecology patients treated with intensity-modulated whole pelvic radiation therapy. Int $\mathbf{J}$ Radiat Oncol Biol Phys 56: 1354-1360, 2003.

16. Mundt AJ, Lujan AE, Rotmensch J, Waggoner SE, Yamada SD, Fleming G and Roeske JC: Intensity-modulated whole pelvic radiotherapy in women with gynecologic malignancies. Int $\mathrm{J}$ Radiat Oncol Biol Phys 52: 1330-1337, 2002.

17. Roeske JC, Lujan A, Rotmensch J, Waggoner SE, Yamada D and Mundt AJ: Intensity-modulated whole pelvic radiation therapy in patients with gynecologic malignancies. Int J Radiat Oncol Biol Phys 48: 1613-1621, 2000.

18. Roeske JC, Bonta D, Mell LK, Lujan AE and Mundt AJ: A dosimetric analysis of acute gastrointestinal toxicity in women receiving intensity-modulated whole-pelvic radiation therapy. Radiother Oncol 69: 201-207, 2003.

19. Kachnic LA and Powell SN: IMRT for breast cancer - balancing outcomes, patient selection, and resource utilization. J Natl Cancer Inst 103: 777-779, 2011.

20. Mayo CS, Urie MM and Fitzgerald TJ: Hybrid IMRT plans - concurrently treating conventional and IMRT beams for improved breast irradiation and reduced planning time. Int $\mathrm{J}$ Radiat Oncol Biol Phys 61: 922-932, 2005.

21. Farace P, Zucca S, Solla I, Fadda G, Durzu S, Porru S, Meleddu G, Deidda MA, Possanzini M, Orrù S and Lay G: Planning hybrid intensity modulated radiation therapy for whole-breast irradiation. Int J Radiat Oncol Biol Phys 84: e115-e122, 2012.

22. Thilmann C, Zabel A, Milker-Zabel S, Schlegel W, Wannenmacher $M$ and Debus $J$ : Number and orientation of beams in inversely planned intensity-modulated radiotherapy of the female breast and the parasternal lymph nodes. Am J Clin Oncol 26: e136-e143, 2003.

23. Van Dyk J (ed): The Modern Technology of Radiation Oncology. Vol 2. Medical Physics Publishing, Madison, WI, 2005.

24. Caudell JJ, De Los Santos JF, Keene KS, Fiveash JB, Wang W, Carlisle JD and Popple R: A dosimetric comparison of electronic compensation, conventional intensity modulated radiotherapy, and tomotherapy in patients with early-stage carcinoma of the left breast. Int J Radiat Oncol Biol Phys 68: 1505-1511, 2007.

25. Ragaz J, Jackson SM, Le N, Plenderleith IH, Spinelli JJ, Basco VE, Wilson KS, Knowling MA, Coppin CM, Paradis M, Coldman AJ and Olivotto IA: Adjuvant radiotherapy and chemotherapy in node-positive premenopausal women with breast cancer. N Engl J Med 337: 956-962, 1997. 
26. Overgaard M, Hansen PS, Overgaard J, Rose C, Andersson M, Bach F, Kjaer M, Gadeberg CC, Mouridsen HT, Jensen MB and Zedeler $\mathrm{K}$ : Postoperative radiotherapy in high-risk premenopausal women with breast cancer who receive adjuvant chemotherapy. Danish Breast Cancer Cooperative Group 82b Trial. N Engl J Med 337: 949-955, 1997.

27. Lo YC, Yasuda G, Fitzgerald TJ and Urie MM: Intensity modulation for breast treatment using static multi-leaf collimators Int J Radiat Oncol Biol Phys 46: 187-194, 2000.

28. Evans PM, Donovan EM, Partridge M, Childs PJ, Convery DJ, Eagle S, Hansen VN, Suter BL and Yarnold JR: The delivery of intensity modulated radiotherapy to the breast using multiple static fields. Radiother Oncol 57: 79-89, 2000.

29. Donovan EM, Johnson U, Shentall G, Evans PM, Neal AJ and Yarnold JR: Evaluation of compensation in breast radiotherapy: a planning study using multiple static fields. Int J Radiat Oncol Biol Phys 46: 671-679, 2000.

30. Zackrisson B, Arevärn $M$ and Karlsson M: Optimized MLC-beam arrangements for tangential breast irradiation. Radiother Oncol 54: 209-212, 2000

31. McDonald MW, Godette KD, Butker EK, Davis LW and Johnstone PA: Long-term outcomes of IMRT for breast cancer: a single-institution cohort analysis. Int J Radiat Oncol Biol Phys 72: 1031-1040, 2008.

32. Morganti AG, Cilla S, Valentini V, Digesu' C, Macchia G, Deodato F, Ferrandina G, Cece MG, Cirocco M, Garganese G, Di Lullo L, Traficante D, Scarabeo F, Panunzi S, De Gaetano A Sallustio G, Cellini N, Sofo L, Piermattei A and Scambia G: Phase I-II studies on accelerated IMRT in breast carcinoma: technical comparison and acute toxicity in 332 patients. Radiother Oncol 90: 86-92, 2009.

33. Chang SX, Deschesne KM, Cullip TJ, Parker SA and Earnhart J: A comparison of different intensity modulation treatment techniques for tangential breast irradiation. Int J Radiat Oncol Biol Phys 45: 1305-1314, 1999.

34. Hong L, Hunt M, Chui C, Spirou S, Forster K, Lee H, Yahalom J, Kutcher GJ and McCormick B: Intensity-modulated tangential beam irradiation of the intact breast. Int $\mathrm{J}$ Radiat Oncol Biol Phys 44: 1155-1164, 1999.

35. Hurkmans CW, Cho BC, Damen E, Zijp L and Mijnheer BJ: Reduction of cardiac and lung complication probabilities after breast irradiation using conformal radiotherapy with or without intensity modulation. Radiother Oncol 62: 163-171, 2002.

36. Fogliata A, Bolsi A and Cozzi L: Critical appraisal of treatment techniques based on conventional photon beams, intensity modulated photon beams and proton beams for therapy of intact breast. Radiother Oncol 62: 137-145, 2002.

37. Brosius FC 3rd, Waller BF and Roberts WC: Radiation heart disease. Analysis of 16 young (aged 15 to 33 years) necropsy patients who received over 3,500 rads to the heart. Am J Med 70: 519-530, 1981.
38. McGale P and Darby SC: Low doses of ionizing radiation and circulatory diseases: a systematic review of the published epidemiological evidence. Radiat Res 163: 247-257, 2005.

39. Preston DL, Shimizu Y, Pierce DA, Suyama A and Mabuchi K Studies of mortality of atomic bomb survivors. Report 13: solid cancer and noncancer disease mortality: 1950-1997. 2003. Radiat Res 178: AV146-AV172, 2012.

40. Ozasa K, Shimizu Y, Suyama A, Kasagi F, Soda M, Grant EJ, Sakata R, Sugiyama H and Kodama K: Studies of the mortality of atomic bomb survivors, Report 14, 1950-2003: an overview of cancer and noncancer diseases. Radiat Res 177: 229-243, 2012.

41. Taylor CW, McGale P and Darby SC: Cardiac risks of breastcancer radiotherapy: a contemporary view. Clin Oncol 18: 236-246, 2006.

42. Mège $\mathrm{A}$, Ziouèche $\mathrm{A}$, Pourel $\mathrm{N}$ and Chauvet $\mathrm{B}$ : Radiation-related heart toxicity. Cancer Radiother 15: 495-503, 2011 (In French).

43. Senkus-Konefka E and Jassem J: Cardiovascular effects of breast cancer radiotherapy. Cancer Treat Rev 33: 578-593, 2007.

44. Darby SC, Cutter DJ, Boerma M, Constine LS, Fajardo LF, Kodama K, Mabuchi K, Marks LB, Mettler FA, Pierce LJ, Trott KR, Yeh ET and Shore RE: Radiation-related heart disease: current knowledge and future prospects. Int J Radiat Oncol Biol Phys 76: 656-665, 2010.

45. Lingos TI, Recht A, Vicini F, Abner A, Silver B and Harris JR: Radiation pneumonitis in breast cancer patients treated with conservative surgery and radiation therapy. Int J Radiat Oncol Biol Phys 21: 355-360, 1991.

46. Lee HK, Vaporciyan AA, Cox JD, Tucker SL, Putnam JB Jr, Ajani JA, Liao Z, Swisher SG, Roth JA, Smythe WR, Walsh GL, Mohan R, Liu HH, Mooring D and Komaki R: Postoperative pulmonary complications after preoperative chemoradiation for esophageal carcinoma: correlation with pulmonary dose-volume histogram parameters. Int J Radiat Oncol Biol Phys 57: 1317-1322, 2003.

47. Graham MV, Purdy JA, Emami B, Harms W, Bosch W, Lockett MA and Perez CA: Clinical dose-volume histogram analysis for pneumonitis after 3D treatment for non-small cell lung cancer (NSCLC). Int J Radiat Oncol Biol Phys 45: 323-329, 1999.

48. Schallenkamp JM, Miller RC, Brinkmann DH, Foote T and Garces YI: Incidence of radiation pneumonitis after thoracic irradiation: dose-volume correlates. Int J Radiat Oncol Biol Phys 67: 410-416, 2007.

49. Chen Y, Thompson W, Semenciw R and Mao Y: Epidemiology of contralateral breast cancer. Cancer Epidemiol Biomarkers Prev 8: 855-861, 1999.

50. Hall EJ and Wuu CS: Radiation-induced second cancers: the impact of 3D-CRT and IMRT. Int J Radiat Oncol Biol Phys 56: 83-88, 2003. 